\title{
KAWSAK SACHA-SELVA VIVIENTE: PERSPECTIVAS RUNA SOBRE CONSERVACIÓN ${ }^{1}$
}

\author{
KAWSAK SACHA-LIVING FOREST: \\ RUNA PERSPECTIVES ON \\ CONSERVATION
}

\author{
Daniel Santi \\ E-mail: urkutulumk@gmail.com \\ FLACSO-Ecuador \\ Marina Ghirotto Santos \\ E-mail: marina.ghirotto@gmail.com \\ PPGAS/USP-Brasil \\ Orcid: https://orcid.org/0000-0001-8220-3327
}

\section{RESUMEN}

Este artículo tiene como objetivo describir y analizar en qué consiste la categoría de "conservación" del territorio del Pueblo Originario Kichwa de Sarayaku, ubicado en la Amazonía ecuatoriana, partiendo de su propuesta denominada Selva Viviente o Kawsak Sacha (en kichwa) y prácticas cotidianas, como la pesca. Aunque esta propuesta haya sido trabajada hace más de diez años, desde 2012 Sarayaku empieza a visibilizarla como parte del esfuerzo por hacer efectivos los derechos de la naturaleza y de los pueblos originarios, previstos en la constitución ecuatoriana de 2008. En julio de 2018, cuando se realizó el evento oficial de lanzamiento de la propuesta en Quito, Sarayaku declaró su territorio como área de protección libre de explotación extractiva, petrolera, minera, maderera, así como de mecanismos de conservación asociados al mercado (como sería el REDD+). Sarayaku enfatizó que esta es una demanda por el reconocimiento jurídico-político de la existencia de otros seres - espíritus, árboles, cascadas, Pachamama o Allpamama, etc. - como vivos y conscientes, al igual que los humanos, a parte de una nueva categoría de conservación de territorios indígenas, ya que la constitución de 2008 y la titulación de su territorio (alcanzada en 1992) no garantiza el mantenimiento de sus prácticas de vida, derechos y tampoco la autonomía en definir lo que es, cómo cuidar e interactuar en/con su territorio. Esta propuesta forma parte de una larga trayectoria, protagonizada por Sarayaku, de lucha y resistencia anti-extractiva, sobre todo petrolera, además de un nuevo marco en la elaboración de conceptos y propuestas (cosmo)políticas que este pueblo ha históricamente presentado al Estado y a las organizaciones internacionales (Naciones Unidas, coaliciones de la sociedad civil, etc.) como un paso más allá de la resistencia "en contra". Si de hecho esta es una traducción al mundo de los no indígenas de conceptos y prácticas concretas de vida de Sarayaku, es también verdad que es más que solamente tácticas o estrategias para el enfrentamiento de la explotación y aniquilación que proviene de los mundos no-indígenas. Constituye, en fin, un modo de vida, una forma de hacer mundo, de resistir co-existiendo.

Palabras-clave: Conservación. Pueblos kichwas. Kawsak sacha. 


\begin{abstract}
The purpose of this article is to describe and analyze the category of "conservation" of the territory of the Kichwa people of Sarayaku, Ecuadorian Amazon, based on their proposal named Living Forest or Kawsak Sacha (in kichwa) and daily practices, such as fishing. Although they have beeen working on this proposal for more than ten years, since 2012 Sarayaku begins to translate it as part of the effort to make the rights of nature and indigenous people effective, both of them ensured by the Ecuadorian constitution of 2008. In July 2018, when the proposal was launched in an official event held in Quito, Sarayaku declares its territory as a protected area free from extractive industry and mechanisms of conservation associated with the market (such as REDD+). Sarayaku emphasized that this is a demand for the legal-political recognition of the existence of other beings - spirits, trees, waterfalls, Pachamama or Allpamama, etc. - as alive and aware, as well as humans, along with a new category of conservation of indigenous territories, since the 2008 constitution and the titling of their territory (reached in 1992) does not guarantee the maintenance of their life practices, rights and autonomy in defining what it is, how to care for and interact in/with its territory. This proposal is part of a long trajectory starred by Sarayaku in fighting against extractive industry, especially oil, as well as a new framework in the elaboration process of concepts and (cosmo)political proposals that this people has historically presented to the State and international organizations (United Nations, coalitions of civil society, etc.) as a step forward to the resistance "against". If in fact this is a translation to the world of the non-indigenous people of Sarayaku's concrete concepts and practices of life, it is also true that it is more than just tactics or strategies for the confrontation of the exploitation and annihilation that comes from the non-indigenous worlds. It constitutes, in short, a way of life, a way of making worlds, of resisting co-existing.
\end{abstract}

Keywords: conservation, kichwa people, living forest.

Figura 1 - Río Bobonaza, territorio de Sarayaku

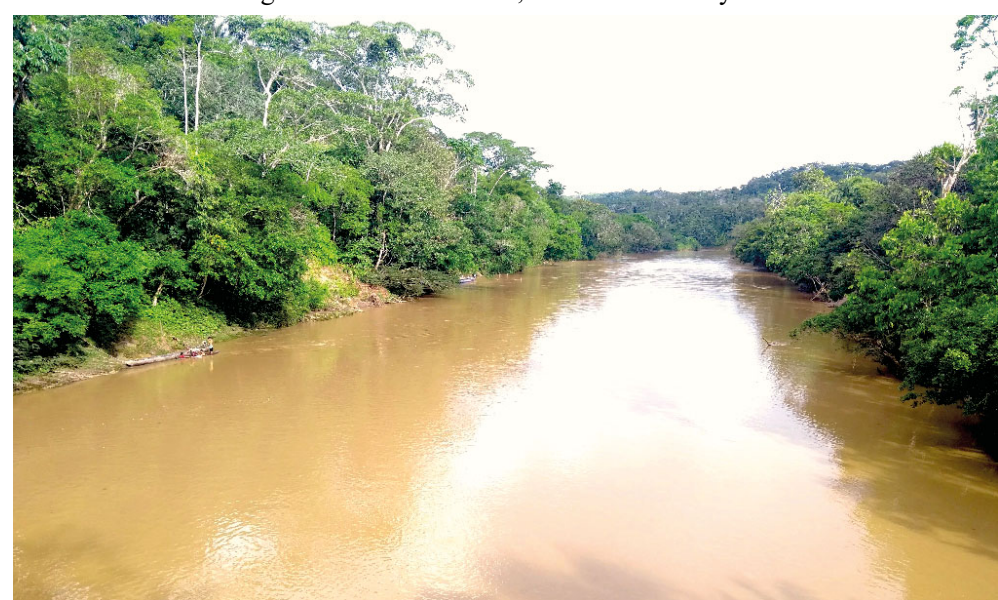

Fonte: Agosto 2018.

\title{
¿PURA SELVA?
}

Era fin de tarde de un viernes en Puyo, capital de la provincia amazónica de Pastaza. José Gualinga, runa ${ }^{2}$ de Sarayaku, y yo, Marina, conversábamos en la sede de su agencia de turismo - Papangu Tour - sobre qué es el Kawsak Sacha-Selva Viviente para él. Sentados en hermosas sillas hechas de madera, vimos entrar en la agencia un sujeto un poco aturdido, con papeles volando por las manos. Dijo él que era belga, que trabajaba para un guía de turismo sobre Ecuador y que él estaba encargado de actualizar los datos acerca de las agencias de turismo en esta región. Él pregunta a José acerca de Papangu y también sobre otras tres agencias que él había calculado existir en Puyo. José, pacientemente, 
le empieza a explicar los lugares en donde su agencia hace visitas - son tres - y el objetivo: no es una agencia común, una vez que hace trabajos con grupos, generalmente de estudiantes foráneos, que vienen a conocer la Amazonía desde una "perspectiva política, de lucha". Sobre Sarayaku, José explica que es un pueblo Kichwa de la Amazonía ecuatoriana, ubicado cerca de una hora en carretera desde Puyo hacia Canelos, y de allí en canoa por el río Bobonaza, por aproximadamente seis horas. Hay también la opción de llegar por avioneta, con la compañía Aero Sarayaku, por ejemplo, saliendo desde la Shell y alcanzando la comunidad en 25 minutos. "En Sarayaku viven cerca de 1.500 personas en siete comunidades”, explica él al señor belga, que contesta: “¿Entonces, no es pura selva, no es cierto?”. Y este añade: “¿Hay agricultura?”, a lo que José responde que, "sí, por supuesto que hay, pero en las áreas determinadas". Con eso el belga concluye que "este es más un tour cultural que natural".

José fue poco a poco - muy despacio, en verdad - introduciendo otras informaciones al señor. De hecho, era muy complejo explicar qué tipo de turismo era ese que Papangu estaba encargada pues implicaba explicar, por ejemplo, una lucha de décadas que Sarayaku viene haciendo en contra de empresas petroleras. Pues bien, José sigue explicando al señor que cuando llevan turistas "hacia dentro" (en la selva, en la comunidad) se realizan charlas y seminarios para hablar de esta lucha del pueblo. Nuevamente, el señor se sorprende: "seminarios en la selva... Entonces Sarayaku en verdad es una organización... ¿Tú eres el presidente de la comunidad?”. José responde que no, pero que ya fue. Luego el señor empieza a preguntar sobre el tour para el Parque Nacional Yasuní ${ }^{3}$. José le explica que allí hay pueblos no contactados. El señor, como si estuviera en éxtasis, insiste en que es imposible que no hayan tenido contacto. Y pregunta: “¿Andan desnudos? ¿No tienen machetes?” No nos creía - en ese punto, yo había hablado por primera vez - que eran pueblos en aislamiento voluntario, o por lo menos no comprendía la misma cosa que nosotros bajo este término.

Aunque podamos imaginar qué sería su perspectiva de "pura selva", de entre otras cosas, al señor belga no tuvo tiempo de hacerle más preguntas para comprenderlo en detalles. Pero la charla fue emblemática para uno fijarse en los malos entendidos que frecuentemente se dan y que suelen ser "productivos" en el sentido de que nos hace pensar (con) - en este caso, de pensar qué es la selva y el territorio para Sarayaku; su relación con la propuesta de la Selva Viviente; qué sería o podría ser una perspectiva runa sobre conservación y por qué esta palabra o concepto está en la punta de la lengua de las personas que viven allí. De hecho, la charla me hacia sentir incómoda; el señor miraba el reloj todo el tiempo y frecuentemente interrumpía a José mientras él intentaba aclarar sus preguntas. Al mismo tiempo, sentía que José no explicaba algunas cosas a él, como si no le importase mucho aclararlas. Mientras eso, el belga creía estar manejando la charla, pero nada más equivocado. José sabia muy bien lo que estaba diciendo y lo que no estaba diciendo. Por más alejada que yo me sentiera del señor belga, me pareció una anécdota de muchos momentos que yo viví cuando trataba de preguntar y comprender algo, pero solo recibía respuestas vagas, inconclusas o simplemente ninguna respuesta. Yo vendría a comprender que, con los runa, para aprender algo uno debe estar preparado para ver (Whitten e Whitten, 2008).

Este artículo es un primer intento de co-autoria entre una mujer brasileña no indígena y estudiante en un programa de doctorado en antropología social, la cual describió el evento arriba, y un hombre runa, estudiante en un programa de post-grado sobre "Liderazgo, Ciudades y Cambio Climático", líder de Sarayaku y coordinador general de la propuesta Kawsak Sacha. Aunque no sea el objetivo de este artículo discutir el proceso de reflexión y escrita conjunta, 
lo que sí es importante señalar es que el texto esta inmiscuido por esta relación: mientras Daniel tiene un doble rol - el de activista defensor de su pueblo y de la propuesta Kawsak Sacha, y el de estudiante en temas que le afecta y importa directamente a su pueblo -, Marina trabaja actualmente con Sarayaku por su tesis. Es decir, es un esfuerzo de pensar y hacer una antropología, una investigación involucrada y descolonizadora, en defensa de derechos (exigencia impuesta incluso por el pueblo), sin alejarse del proceso mismo de reflexión sobre prácticas, sentidos y conceptos que se ponen en movimiento desde esa relación y desde el tema del artículo.

Analizar lo que aquí se propone ha implicado un ejercicio de comparación entre lo que llamaríamos, al principio, concepciones "modernas y/o occidentales", y "runas", y un desplazamiento de las posiciones de "interlocutor/a" e "investigador/a", así como de nativo/a. En muchos momentos, Daniel fue el más interesado en analizar los discursos y las prácticas políticas del mundo no indígena (ahuallacta $)^{4}$, en términos de conservación, mientras Marina intentaba analizar prácticas, eventos o discursos protagonizados por los runa. En síntesis, en muchos momentos Marina fue la nativa para Daniel y Daniel el nativo para Marina, pero la discusión aquí puesta no se encierra solamente en esta relación - incluye otras personas, experiencias, conversaciones e investigaciones que una y otro tuvieron en distintos momentos. Por fin, si sabemos que estas dicotomías son artificiales, su artificialidad no nos parece improductiva. Si las posiciones de "nosotros/as" y "ellos/as" son herramientas, lo que importa es la efectividad y la productividad de ellas, o de la conexión.

\section{¿KAWSANGUICHU $?^{5}$ LA DECLARATORIA KAWSAK SACHA O SELVA VIVIENTE}

Presentada como propuesta política, la declaratória Kawsak Sacha empezó a ser gestada por personas de Sarayaku, desde charlas y vivencias con ancianos/as, apamamaguna (abuelas), apayayaguna (abuelos) y los yachak ${ }^{6}$, ya hace más de diez años. Aunque el "porqué" y el "por quién", en aquel contexto, tiene hoy día múltiples explicaciones, el hecho es que la propuesta se tornó tanto una herramienta de lucha hacia fuera - una propuesta política para que los no indígenas entiendan qué es esta vida que se defiende - como hacia dentro del pueblo de Sarayaku y del movimiento indígena que reúne distintos pueblos amazónicos. Si uno habla con personas que no ocupan puestos de dirigencia en el Consejo de Gobierno de Tayjasaruta ${ }^{7}$, por ejemplo, y que por lo tanto, tienen otras funciones laborales que no solamente llevar adelante la vida organizativa, uno va escuchar hablar del Kawsak Sacha de la misma manera. Eso quiere decir que, mientras esa propuesta haya sido vista - y criticada - como algo simplista y solamente "para afuera", con demasiada participación de los ahuallacta, se encuentra actualmente difundida en el imaginario, en el discurso y en la práctica de las personas de Sarayaku. Además, importa aquí, no solamente lo que se dice y lo que se hace sobre la Selva Viviente, sino también, el proceso y, sobretodo, la reflexión e investigación hecha por el pueblo mismo para tratar de traducir algo tan complejo y aparentemente - sólo aparentemente - abstracto al mundo de los otros.

De manera general, al Kawsak Sacha se lo ve formado por muchos planos. Algunos de ellos podrían ser organizados, como lo han dicho en muchas ocasiones, en términos de (1) un concepto filosófico; (2) una propuesta política; y (3) una práctica cotidiana. Sobre los dos primeros, veamos qué dice la propuesta adoptada en la Asamblea General del Pueblo Originario Kichwa de Sarayaku en diciembre 2012 y actualizada en junio 2018 - preparación para 
el gran evento de lanzamiento que abarcó cinco días en julio de 2018, en la ciudad de Quito ${ }^{8}$.

La propuesta ${ }^{9}$ explica al mundo que el territorio es Kawsak Sacha, un ser vivo con consciencia constituido por todos los seres de la Selva, desde los más infinitesimales hasta los más grandes y supremos. Incluye a todos los seres de los mundos animal, vegetal, mineral, espiritual y cósmico, en intercomunicación con los seres humanos, brindándoles a estos lo necesario para revitalizar sus facetas psicológicas, físicas, espirituales, restableciendo así, la energía, la vida y el equilibrio de los pueblos originarios. Estos seres, desde las menores plantas hasta los más supremos que protegen la selva son personas (runaguna) que habitan las cascadas, las lagunas, los pantanos, las montañas, los ríos, los árboles y otros lugares del territorio; viven en comunidad (llacta) y desarrollan su vida de manera semejante al ser humano. En síntesis, la Selva Viviente es un telar ecológico; el mundo natural es también un mundo social ${ }^{10}$.

Sarayaku reafirma, frecuentemente, que esta visión no es una simple creencia o un ideal conservacionista. Es, por el contrario, un llamado para las personas del mundo a aprender a sentir esa realidad en su más íntimo ser. "Eso sólo será posible si aprendemos a escuchar y a dialogar con esos otros seres que forman parte de una conversación cósmica que va mucho más allá de nosotros humanos", dice el documento (Sarayaku 2018: s/p). Emprender esa conversación formaría las bases para una vida más respetuosa con la Pachamama y sería el fundamento para concebir, construir y difundir el verdadero Buen Vivir (Sumak Kawsay) en nuestro mundo - "un mundo amenazado por una crisis ecológica de magnitud planetaria” (ibíd).

Pero a parte de ser un esfuerzo en compartir su filosofía, su ética, sus formas de hacer mundos y relacionarse con la vida, es también una propuesta política, práctica y pragmática - tal como suelen decir. En este sentido, haciendo hincapié en los respaldos jurídicos nacionales y internacionales, la propuesta es justamente una demanda por el reconocimiento del mismo carácter de sujetos jurídico-políticos conferidos a los seres humanos a otros seres vivientes y por la declaración de los territorios indígenas como libres, en perpetuidad, de proyectos extractivos - petroleros, mineros, madereros, etc. En palabras del documento (ibíd), "el Pueblo Originario Kichwa de Sarayaku declara el territorio de Sarayaku Kawsak Sacha - Selva Viviente, ser vivo y consciente, sujeto de derechos, como lo hemos reconocido ancestralmente los pueblos y nacionalidades originarias ${ }^{11}[\ldots][\mathrm{y}]$ exhorta al gobierno ecuatoriano a que reconozca y legitime el Kawsak Sacha [...]". Además, es también presentada como una nueva categoría de conservación y protección del territorio de Sarayaku y de otros pueblos originarios amazónicos, una vez que el Kawsak Sacha es el proprio territorio. Eso implica actualmente, en lo que llaman de post-lanzamiento (post-julio de 2018), un trabajo de incidencia política hecho en conjunto con abogados/as y antropólogos/as para que la propuesta se torne una ley en Ecuador y pueda generar precedentes jurídicos a nivel nacional e internacional. Sobre todo esto, nos explica Yacu Viteri, actual dirigente de relaciones internacionales:

La espiritualidad está en todos momentos. Está, por ejemplo, en la siembra, en la agricultura, en la cacería, en todos los actos que como seres lo mantenemos [...] o sea, que existe esa armonía, ¿no? Que existe esa vinculación, esa codependencia entre los seres. De esa manera entendemos. Por eso decimos kawsak, algo que vive, algo viviente. Kawsak Sacha, Selva Viviente. Porque en ella habitan seres que también tienen sus espiritualidades, su forma de habitar y por eso planteamos ese concepto como una nueva forma de ver el mundo, pero es importante fundamentar de que también exista una propuesta, un planteamiento como una nueva categoría de conservación. Porque los Estados... 
conservar... o declarar zonas intangibles, ha sido desde una perspectiva económica $[\ldots]$ y eso ha tenido repercusiones muy negativas en territorios indígenas porque todo lo ven, lo planifican desde la posición, desde la perspectiva y la ambición de un Estado. Por eso Sarayaku, en ese caso, se plantea desde una perspectiva propia, autentica, y desde nuestros territorios. Entonces, eso hemos tratado de hacer entender en los eventos locales, internacionales, para que todo tipo de espacios sociales puedan entender. Los académicos, una comunidad, la población, gente intelectual, gente artista, para llegar a toda población. Por eso es importante valernos de muchos lenguajes, para que la gente pueda entender. (Comunicación personal, marzo de 2018) ${ }^{12}$.

La propuesta está enredada a los discursos, conflictos y alternativas frente al cambio climático, es decir, como una nueva categoría de conservación de territorios indigenas que contribuye para mitigar o revertir el mismo cambio climático. Más aún, desde Sarayaku la propuesta tiene que ver con un rotundo rechazo - no compartido por todos los pueblos y nacionalidades amazónicas del Ecuador - a los mercados de carbono como sería el REDD ${ }^{13}$ y otros, como el proyecto Socio Bosque del gobierno ecuatoriano ${ }^{14}$. Mucho de eso está, quizás, conectado a un aprendizaje y articulación política que Sarayaku viene asumiendo como un reto: las relaciones que hace años han sido establecidas con cooperantes, ONGs y la sistemática participación en conferencias internacionales - como las COPs (Conferência de las Partes sobre Cambio Climático).

En agosto del 2018, incluso, lideres y lideresas de Sarayaku estuvieron en California para hablar en contra del mercado de carbono en la Cumbre sobre la Acción Climática. En este contexto, Sarayaku se ha posicionado en contra de lo que llaman de "falsas soluciones" - el mercado de carbono -, una vez que recibir recursos de estos fondos para preservar los bosques amazónicos implica "mercantilizar la naturaleza", deforestar y explotar otros lados del mundo. Sarayaku se encuentra en conflicto directo con el entonces prefecto provincial, que estuvo también en California por los mecanismos de mercado y de los cuales ya recibe recursos para hacer factible la propuesta de conservación denominada "Área Ecológica de Desarrollo Sostenible Provincial" (es decir, en la Provincia de Pastaza) establecida bajo una ordenanza. ¿Cuáles serían las prerrogativas para los territorios que adentran (sin consulta) este mercado? ¿Qué significa conservar desde esa perspectiva? ¿Cuál es la diferencia en relación a la propuesta del Kawsak Sacha?

Una vez que el Área Ecológica está sostenida con recursos provenientes del mercado de carbono; que no se ha hecho la consulta libre, previa y informada a los pueblos y nacionalidades indígenas; y que por lo menos $80 \%$ de los bosques en la provincia de Pastaza son territorios indígenas, Sarayaku visibiliza la propuesta Kawsak Sacha justamente en contra del prefecto y al mismo tiempo como una alternativa. Sarayaku manifiesta que el Kawsak Sacha no es una propuesta de conservación clásica bajo el modelo de la llamada "primera generación", en la cual simplemente se encierran los territorios para obtener recursos económicos. A parte de eso, considera que la ordenanza precisa enfrentar la actividad extractiva, considerando que casi la totalidad del territorio de la Amazonía Sur-Oriente está ya entregado o por concesionar a las empresas extractivas petroleras, irrespetando la sentencia de la Corte Interamericana de Derechos Humanos (CIDH) y otros tratados internacionales ${ }^{15}$. Mirian Cisneros, presidenta del Consejo de Gobierno de Sarayaku, añade en rueda de prensa post-California ${ }^{16}$ :

Rechazando la ordenanza por el prefecto, en este caso el gobierno provincial, no hubo consulta ni socialización. Eso viola nuestros derechos [...] Si quieren trabajar, señor prefecto, que coordinemos! Tenemos nuestras proprias propuestas, como es el Kawsak Sacha. Nuestros ter- 
ritorios no están en venta, no están para negociar, son territorios sagrados. Nosotros no somos un pueblo lleno de ignorantes, como nos trató el coordinador del programa. 2,5 millones de hectáreas son afectadas, en nuestros territorios, no se puede tomar la decisión por nosotros, nosotros también tenemos derecho. [...] Eso es parte de la compensación de carbono donde las industrias extractivas siguen actuando para que nosotros preservemos mientras otros pueblos del mundo están desapareciendo. [...] No queremos que nos paguen para que sigan explotando otro lado.

Conservar, desde la perspectiva o propuesta de Sarayaku, implica también trabajar entre todos los pueblos y nacionalidades un instrumento legal estructurado sobre mecanismos de participación propio y directo en los recursos económicos. Lo que plantea Sarayaku es que, el sistema de financiamiento actual (ONU, otros organismos internacionales y inversiones privadas) no solamente excluye de manera definitiva a los pueblos y nacionalidades originários, sino que también los mismos gobiernos de "países desarrollados", bancos multilaterales y empresas privadas que participan del mercado de carbono son los inversionistas mayores en áreas estratégicas para la explotación de los recursos fósiles a nivel mundial. Sarayaku vislumbra, para un futuro próximo, ser copartícipe en proyectos del Estado que disminuían su dependencia del Estado - como ya sería la empresa comunitária de aviación civil con fines lucrativos, Aero Sarayaku. Por lo tanto, para Sarayaku, autonomía y conservación, hoy en día, está también relacionada a una sostenibilidad monetária de la cual "antes" no se hablaba ( $\tan )$ abiertamente, pero que no debe venir de mecanismos de mercado ni tampoco de explotación extractivista. "De nosotros sólo quieren escuchar la parte romántica, bonita, pero cuando hablamos de dinero nadie nos quiere escuchar", dijo el mismo José Gualinga.

En 2008, el Ecuador aprobó la más reciente constitución en la cual se declaró el Estado como plurinacional e intercultural, así como los derechos de la naturaleza y/o pachamama y el buen vivir/sumak kawsay. Esto ha sido fruto de un proceso de lucha, conceptualización y elaboración de propuestas protagonizado por pueblos indígenas que alcanzó al Estado, en el que la capacidad organizativa de Sarayaku desempeñó un papel fundamental. Pero la evaluación general de este proceso, que ya lleva más de 10 años, es que el gobierno, sobre todo el de Rafael Correa, se ha apropriado y tergiversado los conceptos. En la Amazonía, y Sarayaku no es excepción, los enfrentamientos entre pueblos y nacionalidades frente el Estado y empresas, sobre todo cuando el tema es petroleo y minería en sus territorios (con la justificativa de acabar con la pobreza), siguen vigentes y expresan los alejamientos conceptuales y políticos entre uno y otro (Acosta, 2012 Vallejo, 2014).

En este mismo sentido, lideresas y líderes de Sarayaku han dicho repetidas veces que la propuesta del Kawsak Sacha surge justamente porque la "simple" titulación de los territórios - en el Ecuador, alcanzada en 1992 cuando pueblos de la Amazonía marcharon desde allí hacia Quito (cerca de 240 kilómetros) para reivindicar los derechos de propiedad de sus territorios - no les garantiza, por ejemplo, mantener el petróleo bajo tierra. Es decir, si la demarcación o titulación de los territorios indígenas en Ecuador no les asegura su autodeterminación, la propuesta Kawsak Sacha, aliada a una visibilización del concepto runa de conservación, es un otro "plan" de lucha que busca ir más allá del reconocimiento, del reconomiento "por la mitad" o, enfin, del no reconocimiento por parte del Estado en lo que se refiere a la autonomía y autodeterminación de los pueblos indígenas. Además, para Sarayaku es considerada otro hito historico una vez que, con eso, no solamente "se oponen" o resisten en contra el Estado, pero también proponen, con conceptos y prácticas proprios. 
Bajo esta atmósfera, Sarayaku no sólo transita entre territorios prácticos-conceptuales, como también conoce el mundo de los no indígenas, de los análisis científicos sobre el cambio climático y las alternativas, pensando, interviniendo y proponiendo ante este(s) un (des)acuerdo sobre la propia definición de naturaleza, de política y de humanidad capaz, tal vez, de retardar sus estabilizaciones (De la Cadena, 2015; Stengers, 2014). De forma similar a lo que ha ocurrido anteriormente con los conceptos de plurinacionalidad, sumak kawsay y derechos de la naturaleza, una aproximación a la Selva Viviente bajo esta perspectiva nos permite pensar las políticas indígenas como contra-estado y como contra-poder centralizado (incluyendo sus instituciones de gestión local, nacional y global), al mismo tiempo que establecen un diálogo con ese mismo Estado, pero yendo más allá. Se trata, en fin, no de preguntarnos cómo hacer pueblos indígenas y los "más que humanos"17 entraren en las políticas (modernas), y sí en como agenciamientos runa responden (y construyen) estas políticas de diversas formas $-\mathrm{y}$, al hacerlo, cómo desplazan conceptos y mundos no indígenas ${ }^{18}$.

\section{“AQUI NO NOS VAN A VENIR A ENSEÑAR A HACER LA CHAGRA; ESO YA LO SABEMOS $" 19$}

A parte de las propuestas y pugnas directamente relacionadas a la visibilización de su propia categoría de conservación en oposición a las modernas, occidentales, de mercado, etc., interesa, aquí, analizar la permanente y detallada (auto)reflexividad que ha caracterizado todo el proceso de elaboración de la propuesta del Kawsak Sacha. Como concepto o categoría, explican como fue trabajada, durante varios años, vía un proceso sistemático y proprio de investigación, cuyo objetivo fue el de hacer inteligible - o traducir - algo que, para el pueblo es evidente, pero que para el mundo no indígena no lo es. El profundo y permanente estudio de su mundo, através de los yachak, ancianas y ancianos, y del mundo de los no indígenas, de sus conceptos y políticas en un amplio sentido, reactualiza algo que Whitten y Whitten (2008) ya habían dicho: para los runa ser plenamente humano significa conocer otras culturas (humanas y más que humanas), porque por medio de este conocimiento uno conoce el suyo mismo. Veamos un ejemplo:

¡Primeramente, para un individuo indígena que vive en su proprio territorio, en su propia selva, tenemos un lenguaje adecuado para entender la realidad nuestra y entender lo que es el Kawsak Sacha, lo que significa la selva para nosotros, lo que significa el río, lo que significa las montañas, y es un lenguaje común que también hemos aprendido de los yachaks, grandes conocedores, sabios de nuestra comunidad! [...] Pero en un evento es difícil hacer entender a la gente que no conoce nuestra realidad, que ni siquiera conoce lo que es la Amazonía. Que vive en otra realidad, tiene un otro sentido de lo que es la vida, tienen otra cosmovisión y otras formas de convivir, ¿no? ¡Nosotros tenemos nuestra propia filosofía, nuestra propia cosmovisión, entendemos el mundo desde una cosmovisión, desde nuestra filosofía, lo entendemos porque estamos en el espacio, en el territorio, conviviendo... Entonces allí en los eventos tenemos que valernos de los lenguajes apropiados, puede ser académico, puede ser jurídico, puede ser incluso sociológico, filosófico, para que la gente pueda entender. ¡Entonces se nos ha dificultado un poco, pero hemos avanzado! Por ejemplo, al hablar de territorio de pronto conceptualmente se ha hablado de un espacio geográfico, pero a la vez también cuando hablo de territorio depende del contexto en el cual se está hablando. Ahora, estoy hablando abarcando todo el espacio de vida, me refiero 
a algo integral. De pronto todo ser, o todo individuo de la naturaleza tiene su espacio de vida. Tiene su estructura de vida. Estoy hablando en este caso en términos generales. Y la Pachamama, la Madre Tierra literalmente traducida. Pero para nosotros la Pachamama también... [Para] nosotros los conceptos no son personalizados. O sea, nuestros conceptos abarcan mucho. Si hablo de río, pues, si hablo de agua, en un concepto científico son formulas. Pero para nosotros agua tiene otro sentido porque en el agua están los yakuruna, yakusupais, los seres, yakumama, o sea, ¿hay muchas cosas no? Entonces es por eso que de pronto es interesante en el momento de debatir desde una perspectiva de pueblo o de una perspectiva de comunidad sabemos explicar en qué contexto, de qué manera estoy enfocando el concepto. Y eso es lo que nos limita porque para un ciudadano de la ciudad si hablo de tierra de pronto entiende que es una parcela, que está alambrado con un par de alambre [...] y si hablo de territorio de pronto solo se esta fijando en el mapa, territorio, pero para nosotros aquí ya tiene otras dimensiones. Y es por eso que a veces muy poco nos entienden. Entonces, para un académico tratar de estudiar así personalizando va ser complicado. El idioma mismo: por ejemplo [...] yaku es agua, literalmente. Pero en el contexto yaku es una bebida, yaku puede ser un rio, yaku (Sarayaku, rio de maíz), yaku de la laguna tiene otro sentido, otro contexto. Pero mientras para los científicos y los químicos el yaku es simplemente una formula: ¡H2O y punto! Ahí es cuando... y desde esa perspectiva es difícil que se entiendan. ¡La dialéctica de la selva, que relaciones tiene el agua con la montaña, y la montaña donde están los seres... entonces a un científico el yachak le pone loco! (Comunicación personal, marzo de 2018).

Esta aclaración, también de Yacu, nos remite al concepto de equivocación controlada, propuesto por Eduardo Viveiros de Castro (2004), para pensar la antropología como un medio de reconceptualizar, con la ayuda del perspectivismo amerindio, su procedimiento emblemático: la comparación, o mejor dicho, la comparación de antropologías, una vez que el antropólogo y el nativo están involucrados en operaciones intelectuales directamente comparables. La comparación implica el proceso de traducción de los conceptos de los "nativos" a los términos del aparato conceptual de la antropología; pero traducir es justamente suponer que siempre existe una equivocación en el sentido de comunicarse por diferencias, en lugar de silenciar al otro suponiendo una univocalidad o similitud esencial entre lo que el "otro" y "nosotros" estamos diciendo. La equivocación implicada en todo acto de traducción se convertiría en una operación de diferenciación - una producción de diferencia que conecta los dos discursos en la medida precisa en que no están diciendo lo mismo -, yendo más allá de los homónimos equívocos entre ellos. Sin embargo, una equivocación no es un "fallo de comprensión", sino un fallo de comprensión donde los entendimientos no son necesariamente los mismos; no están relacionados con formas imaginarias de "ver el mundo", sino con mundos reales que están siendo vistos. En resumen, se vuelven una equivocación: puede que el sentido común no sea, en verdad, común. El tema, por lo tanto, no es, por ejemplo, el de saber "cómo ve una cascada el mundo", sino qué mundo se expresa a través de la cascada, de qué mundo es el punto de vista. Si de hecho esta es una lección de la que puede aprender nuestra propia antropología, es también una lección análoga de la propuesta de la Selva Viviente.

\section{RUKUSACHA}

Volvemos ahora a otro plan de reflexión que tiene que ver con las prácticas mismo que se llevan a cabo en el territorio, en la cotidianidad. Entre agosto y septiembre, mes de vacaciones en el Ecuador, las familias (ayllus) se 
mueven hacia las purinas (palabra en kichwa que quiere decir caminata, pero también usada para referirse a un lugar, "las purinas"), sobre todo hacia el río Rotunu, en donde se toma chicha (o aswa, en kichwa), se pesca, se hace cacería, se siembran o cosechan las chagras (huertos, cultivos), se descansa, se disfruta y se hace cerámica. Rukusacha (ruku - ancestro, antiguos; sacha- selva) es la palabra comúnmente utilizada para decir en kichwa "bosque primário", y Rotunu sería el Rukusacha por excelencia. En este agosto, a parte de ya haber mucha gente en Rotunu por las vacaciones, hubo un gran evento de pesca para llevar pescado al XV Congreso de la Nacionalidad Kichwa (en Amazonía) y compartir con los parientes. Así que, a parte de las familias que ya estaban ahí de vacaciones (Sarayaku estaba relativamente vacío por eso) y de las otras que llegaban para disfrutar del resto de vacaciones post-lanzamiento en Quito, mucha gente se había mobilizado para estar en Rotunu por la pesca para el Congreso.

El río Rotunu está ubicado bajando (urayta) el río Bobonaza. Siempre se escucha hablar de este río como el lugar donde están todos los espíritus de los animales, los seres de la selva, los espíritus dueños y de los muertos. Es un lugar sagrado; es el Rukusacha, el lugar del Kawsak Sacha, es el Kawsak Sacha mismo. Además, explicaban que si Rotunu es el Kawsak Sacha, Sarayaku es la ciudad y que Puyo es la metrópoli. ¿Pero entonces estarían ellos de acuerdo con lo que decía el belga turismólogo y con las concepciones de conservación de primera generación? ¿Qué Sarayaku mismo no es, entonces, "pura selva"? ¿Qué sería "pura selva" para ellos/as? ¿Cuál es su relación con la propuesta de conservación bajo el concepto de Kawsak Sacha desde el territorio mismo?

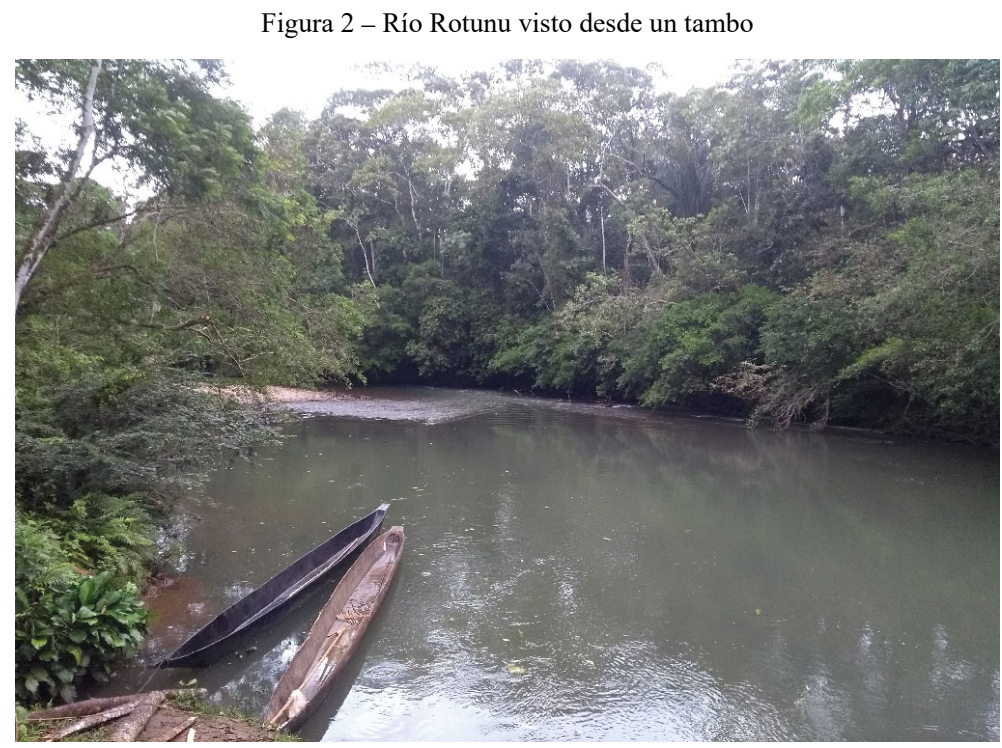

Fonte: Agosto, 2018.

Durante todo el día navegamos por el río Bobonaza y después por Rotunu, río arriba (hanakta). Mientras en la canoa, la presidenta de Tayjasaruta iba indicando los nuevos bloques petroleros, las otras comunidades, donde vivieron, donde pasaban las vacaciones cuando eran niños, donde vivían sus abuelos o donde eran los antiguos tambos - pequeñas construcciones en donde se duerme, se cocina, etc. - de sus antepasados, que hoy en día ya no existen en los mismos lugares. Desde que uno entra en el río Rotunu, se percibe que el ancho es más pequeño, hay más plantas y árboles en el camino, más pájaros, más ruidos. La presidenta estaba muy contenta por estar presentándome al Kawsak Sacha. En un momento, ella y su esposo, que estaba de puntero en la parte frontal de la canoa, discuten quien era más "de la selva". Él dijo que ella pasó su juventud en la ciudad de Quito, mientras ella dijo que creció en Rotunu, 
en plena selva, y que por eso era más de la selva de verdad que él. Finalmente se encerró la discusión, sin consenso; seguimos viaje.

Paramos para visitar y tomar chicha en tres tambos a lo largo del viaje. En el segundo había una radio y, en el, "mientras", una y otra musica; se escuchaban noticias de afuera. La presidenta dice: “¡Música en el Kawsak Sacha!”. Tal como en este momento, ella analizaba y reflexionaba, todo el tiempo, sobre qué era el Kawsak Sacha y hablaba de transformaciones que ella veía allí.

El día siguiente fue el día de la pesca. Nos despertamos bien temprano y fuimos más arriba del río Rotunu. Pasamos por tres redes atrapadoras hechas de bambú. Había muchísimas familias distribuidas en canoas a motor y a remo; en las playas; en los tambos; o en el río mismo (muchos se lanzaban de las canoas al agua, que llegaba hacia la cintura). Con el barbasco los peces se hicieron más fáciles de pescar, así que a las ocho de la mañana ya estaban todos con sus arpones y cestos (ashanga) en manos. Mientras risas y una gran gritaría - “iMira, allí se ve uno!” -, se agarraron algunos. Pero luego vino una lluvia y el nivel del río subió, dificultando la pesca. Los motores se habían colgado. Silencio. Empezamos a bajar nuevamente el río Rotunu. Nos quedamos cerca de la primera red de bambú.

El sol regresó poco a poco y el murmullo de las personas también: casi todas se lanzaron nuevamente al río y había una excitación muy grande en el aire. Algunos peces fueron regalados a la presidenta - que, por su vez, los regalaría cuando regresara a Sarayaku - y otros a la Kurakamama de la comunidad de Kali-Kali, es decir, la esposa del $K_{u r a k a^{20}}$ encargado de organizar la delegación de Sarayaku al Congreso de la Nacionalidad Kichwa. Cuando ya eran cerca de las cuatro de la tarde, paramos en una playa para salar los pescados. Hacía un sol muy fuerte, pero luego vino la lluvia nuevamente. Nos apresuramos a salir y todo el trayecto de regreso - cerca de tres horas hacia el tambo donde dormiríamos nuevamente - fue bajo una fuerte tempestad. La presidenta estaba muy preocupada porque el Kawsak Sacha estaba celoso - celoso de los pescados que habíamos sacado, pero también porque no le gusta gente nueva. Estábamos yo, Marina, el hijo de su esposo, su cuñada y su hijo pequeño - cuatro personas a las cuales el bosque no conocía.

Figura 3 - limpiando los pescados en una playa del río Rotunu

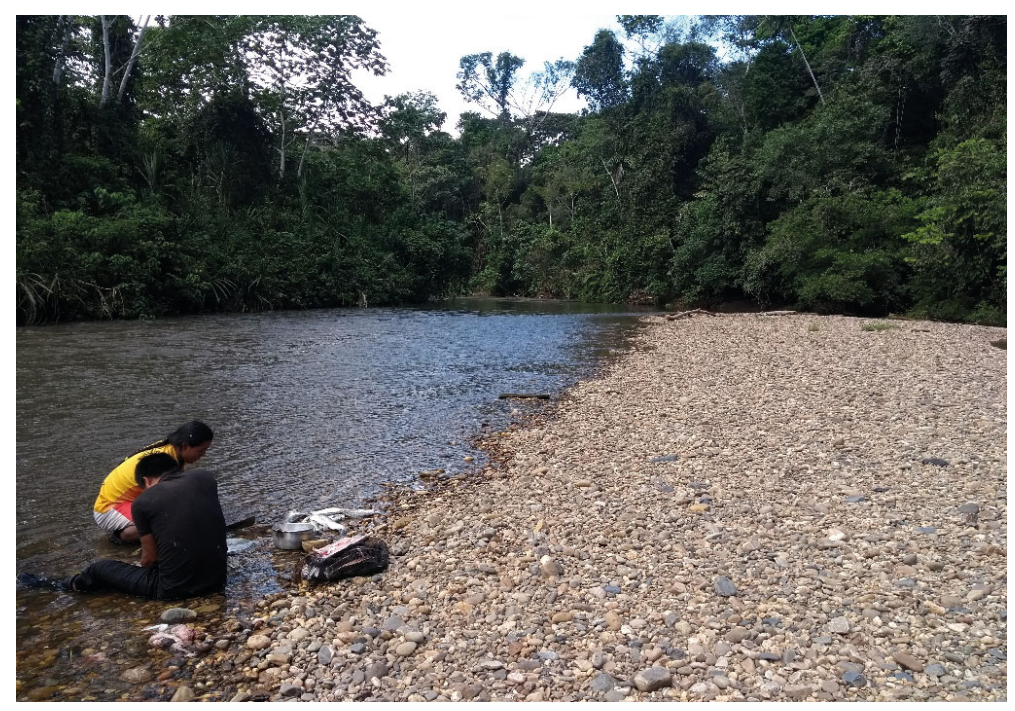

Fonte: Agosto, 2018. 
El esposo de la presidenta me dijo, después de la pesca, que yo debía estar pensando: “¿Pero por qué esos indios sacan tanto pescado? ¿No será demasiado?". Cuando le pregunté porque de hecho sacaban mucho pescado, él me contestó que era por el congreso, para regalar a los otros compañeros kichwas y para las familias de Sarayaku que no se fueron a la purina. A parte de eso, repetidas veces venían a decirme que todo eso ahora era controlado y que no se podría hacer siempre porque, caso contrario, se lo acabaría. Una cierta lógica de abundancia contrasta - y paradoxalmente - se combina con otra de escasez: la selva se va acabar; la abundancia misma se va acabar; los "recursos" son finitos y por eso hay que manejarlos. O sea, como concepto que suele ser abstracto, el Kawsak Sacha es, asimismo, reflexiones y prácticas cotidianas y muy concretas - es decir, la vida misma en el territorio, un territorio que encierra múltiples dimensiones, es hecho a todo momento, y hecho de relaciones.

Es importante fijarse, sin embargo, en la división cosmológica entre selva y chagra - la primera, dominio de los hombres y del espíritu Amazanga; la segunda, de las mujeres y de Nunguli ${ }^{21}$, la dueña de los cultivos -, una división fundante de muchas prácticas y conceptos hoy en día visibilizados por Sarayaku. Si nuestras investigaciones deben partir de una no imposición de nuestras proprias dicotomías en otras ontologías - como serían los pares sexo-género o naturaleza-cultura - eso no quiere decir que algunas dualidades no estén en funcionamento en ese o aquel contexto, aunque no necesariamente las mismas (Mezzenzana 2015). Eso es interesante cuando uno piensa, por qué la propuesta se llama Kawsak Sacha, y no, por ejemplo, Kawsak Chagra: son frecuentes las afirmaciones de que son las mujeres - a parte del yachak - quienes establecen un dialogo con los seres reguladores de uno de los campos más fundamentales de la vida comunitaria - justamente, los cultivos o la chagra. Hay chagras en gran parte del territorio - incluso en parte de las purinas - y es también la Allpamama o Pachamama, una entidad traducida como femenina, la que es visibilizada como la traducción más próxima de lo que sería "la naturaleza" para el mundo no indígena. De todas maneras, cuando uno preguntase qué es la Pachamama, el sacha y la chagra, o por qué del Kawsak Sacha - en el contexto de la propuesta Kawsak Sacha - escucha una respuesta unísona: el sacha es todo, la chagra incluída. Es decir, el sacha no es un bosque sin gente y sin relaciones, ni tampoco un bosque sin cultivos (o cacería, o pesca). El territorio es Sacha Runaguna, es decir, todos los habitantes de la selva, visibles e invisibles, femeninos y masculinos.

Con la categoría de "conflicto entre ontologías" y de "ontología política", la discusión de Blaser (2009) sobre la creación de un programa sostenible de caza entre el pueblo Yshiro del Paraguay nos ayuda a pensar lo que puede estar en juego aquí. Blaser lo piensa como un encuentro entre mundos distintos, donde el "ambiente" puede ser sólo parcialmente lo que está en juego, es decir, algo que importa para algunas partes involucradas en el conflicto, pero para otras, tal vez no estemos seguros. Este "malentendido" también puede encontrarse en el trabajo de Mauro Almeida (2013), que se refiere a una "guerra de ontologías" en el encuentro entre la Caipora, en que el dueño de la caza debe ser respetado para garantizar su disponibilidad, y la ontología económica del mercado, en que sólo lo que se transforma en una mercancía existe o puede existir. Almeida discute como mundos - el de los caucheros, técnicos, científicos y conservacionistas - se encuentran en programas "sostenibles" de caza e involucran diferentes concepciones de lo que existe y de lo que no existe. En esas disputas, Almeida identifica las ontologías - "el acervo de presupuestos sobre lo que existe" - y los encuentros con lo que existe - los "encuentros pragmáticos" - como inseparables. Los presupuestos ontológicos dan sentido y permiten interpretar los encuentros pragmáticos, pero esto también nos permite percibir que es posible tomar otros postulados como datos. 
Desde los runa, conservar el bosque está necesariamente conectado, además, a la gestión de una "propiedad colectiva de la tierra", no solamente bajo los términos de los derechos colectivos de los pueblos indígenas, pero también en el sentido de que todo es habitado y tiene sus seres protectores, sus dueños humanos y más que humanos, aunque quizás no permanentes (una vez que pueden, por ejemplo, cambiar de lugares). Hacemos hincapié en el tema de la dueñidad desde el punto de vista, largamente discutido en la antropología, de que en la Amazonía esta categoría trasciende en mucho la simple expresión de una relación de propriedad o domínio; la categoría designa un modo generalizado de relación, que es constituyente de la socialidad amazónica y caracteriza interacciones entre humanos, entre más que humanos, entre humanos y más que humanos, entre personas y cosas (ver, por ejemplo, Fausto 2008; Cabral, 2012; Matta, 2016).

Cuando una mujer va abrir una nueva chagra, por ejemplo, generalmente tiene la ayuda de su marido u otros parientes hombres, pero debe tener la permisión de Nunguli y de otros dueños/as de los cultivos mismos. En relación a los hombres, las dueñas de las chagras y de los alimentos son, de hecho, las mujeres, que también son las dueñas de la carne del monte o del pescado que traen los hombres. Pero antes, cuando un hombre se va de cacería, tiene que tener el permiso de Amazanga, una vez que este es el dueño de los animales y del sacha, así como de otros innúmeros seres que conviven allí. Yo Daniel, por ejemplo, digo que, cuando uno está en una laguna y dispara un arma, orina o defeca, la laguna se alarma, entonces, comienza a llover y hay un viento fuerte. Los dueños y protectores de la laguna se ponen bravos - ruriruri, yakumama, yakuruna, yakusupay - ¡Y es verdad! He comprobado. En tu casa, si alguien hace eso tú te enojas. Los seres cuidan el entorno. Lo mismo pasa a las piedras grandes, a las cascadas. Eso es difícil de explicar al occidente. Si eres un sabio, eres bienvenido por el bosque; pero una persona desconocida, hasta que se relaciona y conozca, lleva tiempo. Al instante uno se puede enojar o igual conversar en diálogo ${ }^{22}$.

Aún conectado al tema de dueñidad, otro punto que nos parece importante fijarnos en este sentido - y en este artículo - son las reflexiones runas sobre armonía asociadas a la conservación. A diferencia de nociones occidentales de "armonía con la naturaleza" en bosques sin gente y sin dueños, los runa de Sarayaku tienen muy claro qué quieren decir al utilizar esta palabra - aunque, muchas veces, suelen ser mal comprendidos incluso por antropólogos que les dicen "eso [Pachamama, armonía] es una tontería". Cuando preguntado sobre el tema de la armonía con la naturaleza, dice Yacu:

"Nada está puro. Nada, ningún ser, es el centro del problema. Un enfrentamiento hace que tu busques alternativas. Busques soluciones. Y de pronto dentro de la naturaleza también existen actuaciones de ese tipo. Por ejemplo, el hecho de que un animal le ve a otro animal como parte de su dieta, ipues está en conflicto! Pero ese animal que es dieta para otro animal también tiene sus víctimas, y se hace una cadena. Y nosotros cómo uno de los animales, como ser humano, somos tal vez el mayor causante de los conflictos dentro de una naturaleza. Entonces [conflictos] existen. Pero consideramos que si entendemos esta realidad, esos pequeños conflictos, profundos que pueden existir, que pasan por desapercibidos... ¡Pero ahí están los yachaks! Por ejemplo, tenemos muchos mitos donde la actuación del individuo ha hecho que las reacciones de los grandes seres, pues tenga algunas reacciones terribles. Nos han hecho guerra a los seres humanos esos seres protectores. Si tú vas a una laguna, llega y se obscurece, ya estás en conflicto, le estás motivando a que reaccione. Por la noche... y si tú vas sin ir respetando, sin respetar, pues... ya estás en conflicto. Hay reacciones. Entonces ¿qué hacemos los seres humanos? Las actuaciones hay que tener un 
límite para que no se altere. Y eso pasa. Entonces tal vez el concepto de armonía de mi forma de ver no es una armonía pura, una armonía intocable, una armonía que es diseñable, una armonía que es algo así... Una armonía que un día puede desarmarse pero yo pienso que la armonía se va construyendo solamente con la idea de mejorar, de ir comprendiendo al otro pero mejorando las condiciones de existencia, porque si tú estas viendo que vas a destruir el río, que vas a contaminar y conscientemente lo haces, pues... No estás tratando de convivir armónicamente, lo estas destruyendo por destruir, a lo loco. Entonces, pienso que la armonía es también un concepto de construcción, un concepto de entender al otro, y en base a ese entendimiento poder coexistir.'(Comunicación personal, marzo de 2018)

En medio de esta miríada de conceptos, personas humanas y más que humanas, en Sarayaku, igual que en muchos otros pueblos amazónicos, las relaciones de protección y de dueñidad hoy en día están también yuxtapuestas a las zonificaciones previstas en los Planes de Vida (Sumak Kawsay Ñampi). Esos surgieron contra de la explotación petrolera y la nueva era del saqueo con la minería a gran escala, agronegocios, explotación hidrocarburífera no convencional, industria forestal, construcción de megaproyectos infraestructurales (represas, carreteras, termoeléctricas, gasoductos, entre otros). Los pilares del Plan de Vida de Sarayaku son tres: (1) el Sumak Allpa (tierra fértil), que se refiere al ambiente, los recursos naturales y el territorio; (2) el Runaguna Kawsay (vida del pueblo), el eje de la economía solidaria (chagra, pesca, cacería, artesanía y ming $a^{23}$ ), economía monetaria, lo organizativo, lo político, lo social y servicios básicos (infraestructura, caminos vecinales, pista, comunicación) del pueblo; y (3) el Sacha Runa Yachay (conocimiento y sabiduría del pueblo de la selva), que trata de la educación, cultura, ciencia y tecnología propia, salud y medicina tradicional, arte y música, cosmovisión, bio-conocimientos y administración de la justicia indígena.

Sobre el eje Sumak Allpa (Kawsak Sachata Purichina Ñampi o Plan de Gestión Territorial y Protección del Kawsak Sacha $)^{24}$, explican que es el plan de administración y gestión sostenible de los ecosistemas del territorio de Sarayaku, así como el cuidado del Kawsak Sacha: políticas y programas de conservación que se basan en los conocimientos del Sacha Runa Yachay, construido con la participación colectiva de los miembros del pueblo. El Plan implica la zonificación del territorio con criterios de conservación del Sumak Allpa, aplicando normativas de convivencia, monitoreo de la fauna y flora, medición de la pobreza bajo criterios proprios, vigilancia para la protección del territorio, directrices para reforzar la aplicación de técnicas propias de gestión territorial, etc ${ }^{25}$. Como una de las más simbólicas iniciativas está el Sisa Ñampi, el camino viviente de las flores o frontera de vida, un sendero viviente de miles de árboles florísticos y frutales sembrados en anillos cada cuatro kilómetros a lo largo del perímetro del territorio ancestral de Sarayaku, que se puede ver desde el aire. Sisa Nampi es el emblema de paz y de resistencia para la vida, el escudo de conservación de la selva amazónica, la preservación de los lugares vivos y de todos los pueblos y seres que viven allí. A parte de eso, Sarayaku ha construido el jardín botánico llamado "Sacharuya", un proyecto para salvaguardar y reproducir variedades y especies de plantas y semillas de la zona; y otras iniciativas, como el Banco Solidario "Mushuk Kawsay". Son estas directrices que, asociadas a otros modos de conocer y hacer mundos, orientan el control de la pesca en Rotunu, por ejemplo. 


\section{CONSIDERACIONES FINALES}

Pensando con Marisol de la Cadena $(2015 ; 2018)$, en las declaraciones aqui recogidas, tierra y territorio, sacha y chagra, pueden referirse tanto a una porción de tierra bajo la jurisdicción del Estado ecuatoriano cuanto a una entidad que emerge a través de prácticas de vida que pueden, por ejemplo, hacer personas parientes de la tierra, de los ríos, de seres más que humanos es decir, lo que determina el territorio depende del mundo que lo pronuncia, de las relaciones de las que emerge. Esta relación - en la que las personas y el territorio están juntas - sobrepasa (exceed) las posibilidades de los humanos modernos y de la naturaleza moderna, así como las relaciones modernas entre ellos, sin excluirlas. Eso es lo que garantiza la posibilidad del exceso o de "no ser solamento eso" - un "desacuerdo ontológico" que puede ser productivo, pues se refiere a la implementación de mundos en divergencia que pueden tener la capacidad de se conectar parcialmente y afectar la propria política moderna. Por lo tanto, nos parece que hay más en cuestión que "solamente" la defensa del territorio, una protesta contra la expansión capitalista o una preocupación por el destino del medio ambiente. Lo que también está en juego es una concepción y una forma distinta de vida (Povinelli 2016).

En síntesis, el Kawsak Sacha, el territorio de Sarayaku y el Rukusacha son conceptos-entidades a los cuales uno no puede definir con pocas palabras; uno tiene que estar preparado para ver, y asimismo, probablemente, no podrá decir exactamente qué es- o no podrá decirlo afuera de una equivocación en donde los presupuestos se vuelven explícitos. En términos de conservación, mientras el Kawsak Sacha sea presentado como el Rukusacha y este sea traducido como bosque primario, de hecho tiene otro sentido o un sentido más amplio que un bosque sin gente, agricultura, caza y pesca. En verdad, es todo lo contrario: sólo es territorio porque es vivo, tiene gente(s) (humana y más allá que humana), cultivos, relaciones, acciones y contra-reacciones. En este sentido, conservar en Sarayaku tiene que ver con transformaciones, pero en sus proprios términos. Estos términos pueden combinarse o ir en contra de las prácticas del Estado, de empresas y ONGs, pero asimismo van más allá de perspectivas que asocian superficialmente a los indígenas como pueblos que destruyen la naturaleza, que la "conservan naturalmente" y/o pueblos que "impiden el desarrollo" (o, en este caso, la mitigación del cambio climático). El esfuerzo de traducción que conecta conceptos runa y no runa no deja, en fin, de ser una forma de hacer brotar otros "en contra" a la ontología propia del Estado y del capitalismo mismo (Sztutman 2013), así como de proponer reajustes en nuestros proprios vocabularios y mundos- antropológicos o no.

\section{NOTAS}

${ }^{1}$ Una versión preliminar de este artículo fue presentada en la "31a Reunião Brasileira de Antropologia”, en diciembre de 2018, Brasília/DF, Brasil. El artículo tiene el apoyo de la Fundação de Amparo à Pesquisa do Estado de São Paulo (FAPESP) desde la investigación doctoral de Marina Ghirotto Santos (Proceso 2017/17805-2).

${ }^{2}$ Runa es una palabra kichwa para referirse a sí mismo y a otras personas y grupos, hablantes del kichwa, cuya traduccion más próxima sería "gente" en el sentido de persona o grupo. En el Ecuador, los pueblos kichwas de las tierras bajas se dividen en cuatro grupos superpuestos a las provincias en la región amazónica: Napo (Napo Runa, Napo Quichuas o Quijos Kichwa), Orellana (Napo Runa o Quichuas de Napo), Pastaza (Canelos Quichua o Pastaza Runa) y Sucumbíos (Aguarico, Lago Agrio) (Whitten 1976; Reeve, [1988] 2002; Kohn, 2013; Uzendoski, 2010; Chavéz et al, 2005; Hudelson, 1996). Sarayaku pertenecería al grupo Canelos Kichwa; sin embargo, se identifican como "Runaguna" (-guna es el sufijo de plural), "Sarayaku Runaguna" o "Pueblo Autónomo Originario Kichwa de Sarayaku". Aún así, los Canelos Kichwa son aquellos con los que los Sarayaku Runa sienten más afinidad, marcando sus características distinti- 
vas en relación a los Napo Runa del norte de la Amazonía ecuatoriana, así como a los kichwas de las tierras altas andinas, por ejemplo. El idioma de los kichwas amazónicos pertenece a la familia lingüística quechua (también quichua o kichwa). En la Amazonía ecuatoriana, los lingüistas Orr \& Wrisley (1981 [1965]) y también Uzendoski \& Whitten (2014) identifican al menos tres variaciones dialectales: Tena, Loreto-Ávila y Bobonaza-Curaray-Puyo (las dos primeras pertenecen a los Napo Runa y la última a los Canelos Runa). Sarayaku y otros pueblos kichwas amazónicos denominan su idioma Runa Shimi, cuya traducción al castellano sería "lengua de gente", "lenguaje de gente" o, en una traducción más simple, "lengua kichwa". Mas allá de la clasificación de los kichwas amazónicos como "andinos aculturados", tanto en la literatura etnológica y etnohistórica como en la memoria de los ancianos/as, los runa se habrían formado a partir de matrimonios interétnicos entre, principalmente, pueblos de lengua jivaroana y zaparoana que adoptaron el kichwa como lengua franca en el periodo colonial - un proceso impulsado por los misioneros en las tierras bajas de Perú y Ecuador desde el siglo XVI, pero también, y quizás antes, una adaptación de estos pueblos a los efectos de la invasión europea.

${ }^{3}$ El Parque Nacional Yasuní, considerado la zona más biodiversa del planeta, se extiende sobre un área de aproximadamente 10.200 kilómetros cuadrados en las provincias de Pastaza y Orellana, entre el río Napo y el

Río Curaray. Fue designado por la Unesco en 1989 como una reserva de la biosfera y es parte del territorio donde se encuentra ubicada la nacionalidad Waorani y dos pueblos en aislamiento voluntario, los Tagaeri y Taromenane. En 2013, el proyecto Yasuni-ITT - cuyo objetivo fue dejar el petróleo existente en el Parque bajo tierra en cambio de una compensación financiera internacional - fue abandonado por el gobierno de Rafael Correa.

${ }^{4}$ Literalmente, personas o pueblos de las tierras altas (- ahua, que significa "arriba"). El término designa mestizos, blancos, europeos, etc. pero no la población indígena de los Andes.

${ }^{5}$ Kawsanguichu es una expresión para saludar. De forma general es traducida como “¿estás bien?”, “ ¿todo bien?”, pero quiere decir, literalmente, “¿estás vivo?”, a la cual generalmente se contesta "kawsaynimi”, “jestoy vivo!”, “¡estoy aquí!” (kawsana significa "vivir", "estar bien"; -ngi significa "nosotros", mientras -chu es una partícula que se añade en la pregunta cuando se exige una respuesta positiva o negativa).

${ }^{6}$ Yachak significa literalmente "aquel que sabe, que conoce". En Sarayaku, el chaman es yachak (quien viaja a otros mundos con la ayahuasca, por ejemplo), pero no todos los yachaks son chamanes. De todas maneras, "chaman" es una palabra occidental, así que la traducción más adecuada sería de hecho "yachak".

7 Tayjasaruta es un acrónimo que designa al Consejo de Gobierno. La traducción es: "Ta" de tayak, ancestros, antiguos, o los primeros hombres y mujeres del pueblo kichwa de Sarayaku; "Y" de yuyayta, conocimiento, sabiduría y fortaleza espiritual para construir la armonía entre los ayllus (familias) y la naturaleza (Sumak Kawsay/ Buen Vivir); "Ja" de Jatachi, aquel que se levanta para construir el camino de la identidad cultural; "Sa" de Sarayaku, nombre del pueblo, literalmente: río de maíz, o pueblo del mediodía; "Ru" de runaguna, traducido como personas con ética y principios de cultura; "Ta" de Tandanakuy, fuerte unidad para la defensa de los derechos de los pueblos indígenas.

${ }^{8}$ Un gran pabellón en el Parque Arbolito, en la capital Quito, fue ocupado con la vida de Sarayaku: canoas, medicinas, artes, conciertos, cerámicas, una casa kichwa, maitos, fotografías, videos, discursos. Estuvieron presentes más de 200 personas de Sarayaku y inúmeros interesados/as que por ahí han pasado.

9 Texto cuya firma es el "Pueblo Originario Kichwa de Sarayaku" y el "Consejo de Gobierno de Tayjasaruta". El documento presentado en Quito fue escrito en castellano y después traducido al kichwa. Hubo un equipo responsable para escribir el documento con el apoyo de antropólogos y otros parceros de Sarayaku (ver, por ejemplo, el texto de 2016 donde Eduardo Kohn habla de su contribución). El documento ha sido compartido, discutido y consensuado por el pueblo de Sarayaku y expuesto a nivel nacional e internacional.

${ }^{10}$ Por supuesto, lo que explica Sarayaku nos remite a los trabajos de Descola (2015), Latour (2013), Viveiros de Castro (2015), Kohn (2013) y otros que discuten, cada uno a su manera, la infertilidad de la separación moderna entre naturaleza y cultura en el sentido de que esta no funciona en todo lugar - o, por lo menos, no de la misma forma en todos los lugares. 
${ }^{11} \mathrm{La}$ "nacionalidad indígena", en Ecuador, agrupa distintos pueblos desde una lógica nativa de fusión y fisión de pueblos heterogéneos. Elaborada entre las décadas de 1970 y 1980, la "nacionalidad indígena" ha representado una transición cualitativa en relación a la de etnia, esta percibida como una inclusión subalternizada, desde la lógica del Estado, incapaz de desplazar el patrón referente blanco-mestizo de la nación ecuatoriana. Con la constitución de 2008 , se da el reconocimiento de catorce nacionalidades indígenas (de las cuales nueve están en la Amazonía) formadas por un total de dieciocho pueblos.

${ }^{12}$ Los extractos citados como "Comunicación Personal" corresponden a conversaciones registradas por Marina.

${ }^{13}$ Programa de colaboración de las Naciones Unidas para la Reducción de Emisiones de la Deforestación y la Degradación de los bosques en los países en desarrollo (Programa ONU-REDD). El cometido de REDD+ es estimular a los países en desarrollo para que contribuyan a los esfuerzos de mitigación del cambio climático mediante: i) la reducción de las emisiones de gases de efecto invernadero (GEI) con la ralentización, detención y reversión de la pérdida y degradación de los bosques; y ii) el aumento de las remociones de GEI de la atmósfera terrestre por medio de la conservación, gestión y expansión de los bosques (ver sítio de la FAO: http://www.fao.org/redd/initiatives/ un-redd/es/).

${ }^{14}$ El Socio Bosque no sólo intenta compensar financieramente las prácticas de conservación bajo el criterio del Estado pero también erradicar una pobreza muchas veces inexistente. Sobre esto ver, por ejemplo, el sítio del Ministerio del Medio Ambiente del Ecuador y, desde otro punto de vista, la tesis de Carmen Seco Perez (2015).

${ }^{15}$ En el juicio del caso Pueblo Indígena Kichwa de Sarayaku vs. Ecuador (2012), en la Corte Interamericana de Derechos Humanos (CIDH), el gobierno ecuatoriano fue culpabilizado por haber otorgado la concesión para la exploración de hidrocarburos y petróleo (con la utilización de pentolita) a una empresa privada (CGC) en parte del territorio de Sarayaku sin su consentimiento. El proyecto causó, entre otros daños, la contaminación del suelo y las aguas y amenazas a los líderes del pueblo. Ver sentencia de la CIDH sobre el Caso Sarayaku-CGC, 2012.

${ }^{16}$ Vídeo disponible en la página "Sarayaku Defensores de la Selva" en Facebook, día 17 de septiembre de 2018.

${ }^{17}$ El término "no humanos" es criticado porque presupone una negativa desde un punto de referencia - los humanos. Por no habermos llegado a otro más adecuado hasta ahora, mantenemos el término "más que humanos".

${ }^{18}$ Aquí podríamos reflexionar también con la idea de autoantropologia bajo los términos de Strathern (2014) que aleja la definición de "casa" (home) del antropólogo basada en la noción de familiaridad en relación al grupo con el que se trabaja. Esta se daría, en cambio, caso la manera de auto-interpretación del grupo estudiado fuera la propia antropología - o, en otros términos, cuando los sujetos investigados se pensan en los mismos términos que la antropología los piensa y piensa a sí, es decir, a través de conceptos como "sociedad", "cultura", etc.--; y con la de antropología reversa (Wagner, 2009), una vez que ha implicado el estudio - o simetrización- de los esquemas mentales, conceptuales y políticos del mundo no indígena por los Runa mismo - lo que nos lleva, por su vez, a reflexionar sobre las relaciones entre antropólogo y nativo al tomar sus procedimientos como equivalentes y, por consiguiente, reconocer como la propia posición de observador y observado puede ser, en los términos de Roy Wagner, "reversa".

${ }^{19}$ Ponencia de la presidenta de Tayjasaruta Mirian Cisneros en la misma rueda de prensa (opcit, p. 10), refiriendóse a proyectos denominados "agroflorestales" o "agrocultivos" para pueblos indígenas.

${ }^{20}$ Los Kurakas son autoridades ancestrales que actuan en el ámbito de los ayllus (famílias ampliadas), es decir, en cada comunidad que conforma el pueblo (llacta) de Sarayaku.

${ }^{21}$ En la literatura de la región, los nombres de espíritos femeninos han sido descritos de manera distinta. Whitten (1985), trabajando en Puyo, menciona el término Nunghui para designar tanto la Chacra Mama cuanto a la Allpa Manca Mama (o Mangallpa Mama), la mama y/o dueña del barro. Los kichwas de Canelos usualmente utilizan estos dos últimos nombres (Guzmán 1997), mientras que en Sarayaku también usan Nunguli. Esta palabra parece ser una adaptación kichwa al contacto con personas hablantes de lenguas jivaroanas (Descola 1988). 
${ }^{22}$ Aunque no sea el tema de este artículo, aquí es interesante fijarse en la traducción propuesta para el término "política" em kichwa. Por lo general, cuando uno pregunta acerca de eso recibe como respuesta una expresión de duda, silêncios. "No hay; es una palabra muy técnica, muy occidental", dijo un dirigente. Después de pensar, y de forma semejante a otras personas - incluso yo, Daniel -, ha dicho: "Hatun Kuynta(n)aku" o "Hatun Yuyakuna[guna] Kwintanakuy". Yuyai fue traducido como conocimientos, pero el término yuyakuna también podría ser traducido literalmente como política. Hatun es grande e/ou importante, y kwintanakuy sería dialogar, conversar. Política como conocimiento o una gran/importante reunión de conversatório/diálogo. Por lo tanto, política - o diálogo, o conocimiento - no se hace solamente entre seres humanos pero también con seres más que humanos.

${ }^{23}$ Trabajo colectivo a cambio de comida y chicha (cerveza de yuca), también llamado de fiesta.

${ }^{24}$ Una mirada detenida sobre este y no otro eje del plan cuando el tema es conservación es, incluso, definida por el pueblo mismo. Eso no quiere decir, sin embargo, que no esté conectado a los otros ejes.

${ }^{25}$ Desde 2013, para reforzar el plan de gestión territorial, se conformó un equipo de ocho kaskirunaguna (Guardianes de la Selva Viviente) que tienen como funciones principales vigilar todo el territorio para que agentes externos o internos no realicen actividades contaminantes y destructoras dentro del territorio; monitorear periódicamente la zona de Kawsak Sacha; socializar, cumplir y hacer cumplir las normativas de convivencia para el Sumak Kawsay en lo que se refiere a la cacería, pesca, estadía en los tambos y cuidado de las especies en peligro de extinción; proteger los puntos de vigilancia de Sisa Nampi (Camino de Flores); mantener continuamente actualizada la base de datos de la diversidad biológica, la vida de Kawsak Sacha y el movimiento y manejo del territorio. Bajo el mandato de la Asamblea y baseandose en una planificación realizada de manera participativa con el equipo técnico y los dirigentes de Sarayaku, los kaskirunaguna establecen sus expediciones al menos tres veces al mes en todo el territorio de Sarayaku, con el fin de cumplir y dar seguimiento a actividades que están a su cargo. Sus trabajos y resultados se evaluan en las reuniones del Consejo de Gobierno y Asambleas del pueblo.

\section{REFERÊNCIAS}

ACOSTA, Alberto. Buen Vivir, Sumak Kawsay. Una oportunidad para imaginar otros mundos. Quito: AbyaYala, 2012.

ALMEIDA, Mauro. Caipora e outros conflitos ontológicos. R@U Revista de Antropologia da UFSCar. v. 1, p. 7-28, 2013.

BLASER, Mario. La ontología política de un programa de caza sustentable. World Anthropologies Network (WAN)/ Red de Antropologías del Mundo (RAM), n. 4, 2009.

CABRAL, Joana. Entre Plantas e Palavrasișị:Modos de Constituição de Saberes entre os Wajãpi (AP). Tesis de Doctorado - Programa de Postgrado en Antropología Social, Departamento de Antropología de la Faculdade de Filosofia, Letras e Ciências Humanas, Universidade de São Paulo, São Paulo, 2012.

CHÁVEZ, Gina; ROMMEL, Lara; MORENO, María. Sarayaku: el pueblo del cenit. Identidad y construcción étnica informe antropológico-jurídico sobre los impactos sociales y culturales de la presencia de la Compañía CGC en Sarayaku. Quito: FLACSO/CDES, 2005.

CIDH. Pueblo indígena kichwa de Sarayaku vs. Ecuador. Sentencia de 27 de junio de 2012. Disponible en: http://www.corteidh.or.cr/docs/casos/articulos/ seriec_245_esp.pdf. Aceso: 14 ago. 2019.

DE LA CADENA, Marisol. Earth Beings: Ecologies of Practice across Andean Worlds. Durham: Duke University Press, 2015.

. Natureza incomum: histórias do antropo-cego. Revista do Instituto de Estudos Brasileiros, n. 69, 2018. 
DESCOLA, Philippe. La Selva Culta. Simbolismo y Praxis en la ecología de los Achuar. Quito: AbyaYala-IFEA, 1988.

. Par-de là nature et culture. Canadá: Folio Essais, 2015.

FAUSTO, Carlos. Donos demais: maestria e domínio na Amazônia. Mana, v. 14/2, p. 329-366, 2008.

GUZMÁN, Maria Antonieta. Para que la yuca beba nuestra sangre. Trabajo, género y parentesco en una comunidad kichwa de la Amazonía Ecuatoriana. Quito: AbyaYala/CEDIME, 1997.

HUDELSON, John. La cultura quichua de transición. Su expansión y desarrollo en el Alto Amazonas. Quito e Guayaquil: Museu Antropológico/Banco Central/ Abya Yala, 1987.

KOHN, Eduardo. How Forests Think: Toward an Anthropology Beyond the Human. California: University of California Press, 2013.

. Ecopolitics. Lexicon for an Anthropocene Yet Unseen: Theorizing the Contemporary. Cultural Anthropology Website, 2016. Disponible en: https:// culanth.org/fieldsights/796- ecopolitics. Aceso: 3 ene. 2019.

LATOUR, Bruno. Jamais fomos modernos. São Paulo: Editora 34, 2013.

MATTA, Priscila. Modos ameríndios de conhecer as florestas: produção de relações e percepções (versão corrigida). Tesis de Doctorado - Programa de Postgrado en Antropología Social, Departamento de Antropología de la Facultad de Filosofia, Letras y Ciencias Humanas, Universidad de São Paulo, São Paulo, 2016.

MEZZENZANA, Francesca. Living through forms: similarity, knowledge and gender among the Pastaza Runa (Ecuadorian Amazon). Tesis de Doctorado Departamento de Antropología, London School of Economics and Political Science, Londres, 2015.

ORR, Caroline; WRISLEY, Betsy. Vocabulario Quichua del Oriente del Ecuador. Quito: Instituto Linguístico de Verano/Ministerio de Educación Pública, [1965] 1981.

POVINELLI, Elizabeth. Geontologies. A requiem to late liberalism. Durham/ Londres: Duke University Press, 2016.

REEVE, Mary-Elizabeth. Los Quichua de Curaray: el proceso de formación de la identidad. Quito: Abya-Yala, [1988] 2002.

SARAYAKU. Declaración Kawsak Sacha - Selva Viviente, Ser Vivo y Conciente, Sujeto de Derechos. Sarayaku/Quito, 2018.

SECO PÉREZ, Carmen. Sumak Kawsay: Listening to the voices of the Living Forest: Resilience and Identity for Indigenous peoples in the Ecuadorian Amazon. Tesis de maestría - Faculty of Science, Stockholm Resilience Centre, Stockholm University, 2015.

STENGERS, Isabelle. La propuesta cosmopolítica. Revista Pléyade. Centro de Análisis e Investigación Política [CAIP]. n. 14, p. 17-41, 2014.

STRATHERN, Marilyn. Os limites da autoantropologia. In: STRATHERN, Marilyn. O efeito etnográfico e outros ensaios. São Paulo: Cosac Naify, 2014.

SZTUTMAN, Renato. Metamorfoses do Contra-Estado. Pierre Clastres e as Políticas Ameríndias. Ponto Urbe: Revista do Núcleo de Antropologia Urbana da USP. São Paulo, n. 13, 2013.

UZENDOSKI, Michael. The Napo Runa of Amazonian Ecuador. Quito: Abya Yala, 2010.

UZENDOSKI, Michael; WHITTEN, Norman E. From 'Acculturated Indians' to 'DynamicAmazonian Quichua-Speaking Peoples'. Tipití: Journal of the Society for the Anthropology of Lowland South America. v. 12, p. 1-20, 2014. 
VALLEJO, Ivette. Petróleo, desarrollo y naturaleza. Aproximaciones a un escenario de ampliación de las fronteras extractivas hacia la Amazonía suroriente en el Ecuador. Anthropológica. Quito, ano XXXII, n. 32, 2014.

VIVEIROS DE CASTRO, Eduardo. Perspectival Anthropology and the Method of Controlled Equivocation. In: Tipiti: Journal of the Society for the Anthropology of Lowland South America, v. 2, 2004 Disponible en: http://digitalcommons.trinity.edu/tipiti/vol2/iss1/1. Aceso: 13 ene. 2019.

. Metafisicas canibais. São Paulo: CosacNaify, 2015.

WAGNER, Roy. A invenção da cultura. São Paulo: CosacNaify, 2009.

WHITTEN, Norman E. Sacha Runa: Ethnicity and Adaptation of Ecuadorian Jungle Quichua. Urbana: University of Illinois Press, 1976.

WHITTEN, Norman E. Sicuanga Runa, The other side of development in Amazonian Ecuador. University of Illinois, Urbana, 1985.

WHITTEN, Norman E.; WHITTEN, Dorothea Scott. Puyo Runa: Imagery and Power in Modern Amazonia. Chicago: University of Illinois Press, 2008. 